\title{
Factores que influyen en la intención de emprendi- miento del emprendedor híbrido
}

Factors that influence the entrepreneurial intention of the hybrid entrepreneur

\author{
Patricia Gonzales Peralta \\ ESAN \\ pgonza@esan.edu.pe
}


A partir de la teoría del comportamiento planeado, se proponen factores que influirían en la intención de emprendimiento en la segunda etapa del emprendimiento híbrido. Esta investigación conceptual hace uso de información secundaria para el análisis temático de la literatura. De esta manera, se establecen un modelo conceptual y proposiciones. Como resultado, se determinan cuatro factores que influenciarían dicha intención: la expectativa de crecimiento empresarial, el capital social emprendedor, el compromiso organizacional con el empleador y la autoeficacia emprendedora

Finalmente, el estudio tiene tres contribuciones. Primero, ofrece un enfoque multidisciplinario al aplicar la teoría del comportamiento planeado en un nuevo contexto; segundo, plantea proposiciones en un nuevo modelo conceptual para su evaluación empírica posterior; y, tercero, promueve la visibilidad del emprendedor híbrido en la literatura.

Palabras clave: emprendimiento híbrido, teoría del comportamiento planeado, intención de emprendimiento, renuncia al empleo, fomento al emprendimiento

From the theory of planned behavior, factors are proposed that would influence the intention of entrepreneurship in the second stage of hybrid entrepreneurship. This conceptual research makes use of secondary information for thematic analysis of the literature. In this way, a conceptual model and propositions are established. As a result, four factors that influence this intention are determined: The expectation of business growth, entrepreneurial social capital, organizational commitment with the employer and entrepreneurial self-efficacy.

Finally, the study has three contributions. First, it offers a multidisciplinary approach by applying the theory in a new context; second, it offers propositions related to the hypotheses of a new conceptual model for subsequent empirical evaluation; and, third, it promotes the visibility of the hybrid entrepreneur in the public policy literature in support of entrepreneurship.

Keywords: hybrid entrepreneurship, theory of planned behavior, entrepreneurship intention, resignation of employment, promotion of entrepreneurship 


\section{Introducción}

Si bien la literatura no presenta consenso sobre la definición, para los propósitos de esta investigación la intención de emprendimiento se entenderá como la probabilidad subjetiva que atribuye un individuo a establecer un negocio en los próximos cinco años, tras realizar una ponderación de sus creencias relacionadas a esta actividad (Krueger et al., 2000). Esta intención, que precede al comportamiento, se presenta a lo largo del proceso emprendedor establecido a partir de investigaciones del Global Entrepreneurship Monitor y puede variar en intensidad o cambiar con el tiempo en cada una de las etapas de este proceso: desde el emprendimiento latente, naciente o joven hasta el logro de la empresa establecida (Van der Zwan \& Thurik, 2017; Wagner, 2004).

En línea con lo anterior, la intención de emprendimiento se ha estudiado en diferentes contextos, entendiéndose grupos de individuos tales como académicos, estudiantes y empleados del sector de alta tecnología, entre otros (Fernandez-Pérez et al., 2015; Sun et al., 2019). No obstante, las motivaciones que influyen en esta intención podrían ser diferentes entre los distintos grupos, pues algunos individuos se encuentran desempleados y no disponen de otras alternativas laborales, mientras que otros disponen de empleos a tiempo parcial o completo que no satisfacen sus necesidades o expectativas (Bögenhold, 2018). En este sentido, en la literatura de emprendimiento aún se sigue investigando la intención de renunciar al empleo dependiente para dedicarse principalmente al emprendimiento; es decir, la transición al emprendimiento a tiempo completo en el contexto del empleado dependiente (Sørensen \& Fassiotto, 2011).

La literatura muestra que los emprendedores no constituyen un grupo homogéneo pues gran parte de estos mantiene un empleo dependiente $y$, al mismo tiempo, gestiona un nuevo negocio propio (Burke et al., 2008). Estos individuos, también llamados "emprendedores híbridos», realizan esfuerzos de emprendimiento, por lo cual se les considera dentro de la etapa de emprendimiento naciente del proceso emprendedor $y$, eventualmente, se deciden por renunciar al empleo y dedicarse sobre todo a su proyecto empresarial (Viljamaa \& Varamäki, 2017), trayendo con ello beneficios para la sociedad y la economía (Raffie \& Feng, 2014).

Cabe resaltar que este subgrupo de emprendedores se trata de un grupo distinto al de los emprendedores tradicionales, pues estos individuos no se dedican por completo al negocio ni suelen recibir la mayor parte de sus ingresos de este. Asimismo, este subgrupo de emprendedores tampoco debería ser estudiado como grupo de empleados, puesto que estos individuos ya tienen información relacionada a la experiencia de su negocio. Por tanto, se reconoce que los emprendedores híbridos podrían contar con diferentes características que influyen en su intención de emprendimiento (Folta et al., 2010).

El proceso de transición de empleado a emprendedor híbrido como punto intermedio hacia el emprendimiento a tiempo completo ha sido insuficientemente estudiado, pero ha mostrado ser de gran relevancia en la literatura (Ferreira, 2020). Como parte de la primera etapa de este proceso, se ha estudiado la intención de emprendimiento que conduce a la transición de empleado a emprendedor híbrido a partir de expectativas de beneficios monetarios y no monetarios (Block \& Landgraf, 2016), utilizando también como marco teórico la teoría del comportamiento planeado (Farooq \& Talib, 2019). Asimismo, 
en la segunda etapa del proceso, a pesar de la relevancia del tema, la literatura muestra un número reducido de investigaciones que estudian la intención de emprendimiento que conduce a la transición de emprendedor híbrido a emprendedor a tiempo completo, utilizando modelos de intención apropiados (Luc et al., 2018). En este sentido, surge la pregunta en torno a cuáles son los principales factores que influenciarían la intención de emprendimiento en el emprendedor híbrido.

El objetivo de la presente investigación es identificar los recién mencionados factores principales que influencian la intención de emprendimiento en el emprendedor híbrido. El problema de investigación presentado es relevante, dado que contribuye a llenar un vacío teórico en cuanto a los modelos de intención de emprendimiento en la segunda etapa del emprendimiento híbrido, en la que el emprendedor renuncia al empleo para dedicarse a emprender a tiempo completo (Thorgren et al., 2016). Asimismo, aporta de manera general a la teoría del comportamiento planeado al aplicarse en un contexto novedoso y promueve la visibilidad del emprendedor híbrido en la literatura que fomenta las políticas públicas. Además, establece un nuevo modelo conceptual, ofreciendo proposiciones relacionadas para una evaluación empírica posterior.

Este conocimiento permitiría comprender con mayor profundidad las principales dinámicas que influyen en la generación de la actividad económica temprana, en específico los negocios que parten de emprendedores híbridos que, como ha sido evidenciado en la literatura, sobreviven más tiempo que otros negocios y son más receptivos a los beneficios de los programas de apoyo al emprendimiento (Schulz et al., 2016; Raffie \& Feng, 2014).

La siguiente sección presenta el marco teórico que sirve como guía para enfocar el emprendimiento híbrido. Luego, los cuatro factores que influirían en la intención de emprendimiento de emprendedores híbridos - la expectativa de crecimiento empresarial, el capital social emprendedor, el compromiso organizacional con el empleador y la autoeficacia emprendedora- serán introducidos para presentar el modelo conceptual. Finalmente, se presentarán la discusión, las implicaciones y las limitaciones del estudio, continuando con las conclusiones y recomendaciones como futuras líneas de investigación.

\section{La teoría del comportamiento planeado}

Según Miner y Dachler (1973), las teorías generales sobre la motivación humana se pueden clasificar en dos grupos: las teorías de contenido y las teorías de proceso. Las teorías de contenido buscan explicar los elementos inherentes al ser humano que causan, mantienen o detienen un comportamiento, y se concentran principalmente en las necesidades del individuo como fuentes de motivación. Por otro lado, las teorías de proceso buscan explicar los procesos cognitivos que conducen a un determinado comportamiento a partir de los factores motivacionales. Desde el punto de vista de los procesos, el interés por la predicción del comportamiento surge a partir de la teoría de expectativa-valor (Atkinson, 1964; Vroom, 1964), que propone que la elección de los individuos, la persistencia y el desempeño pueden ser explicados por sus creencias, ya que el valor atribuido a un comportamiento es estimado por la probabilidad percibida de realizar el comportamiento exitosamente y obtener utilidad de ello. 
Ante la complejidad que implica validar los modelos de expectativa-valor en la literatura, y con el objetivo de predecir el comportamiento de la manera más simple y eficiente, se plantea la teoría de la acción razonada (TAR) (Fishbein \& Ajzen, 1975) o teoría del comportamiento planeado (TCP) (Fishbein \& Ajzen, 2010), que propone la agregación de grupos de creencias en actitudes o disposiciones generales que, en conjunto, determinan la intención de realizar el comportamiento. Adicionalmente, la intención es definida como la probabilidad subjetiva que asigna el individuo respecto a la realización de un comportamiento al ubicarse en una situación hipotética.

Según Ajzen (1991), las tres principales clasificaciones de creencias son actitud general, norma subjetiva y percepción de control sobre el comportamiento. El primer grupo se relaciona con las consecuencias específicas positivas o negativas de realizar el comportamiento, creencias que son más fuertes mientras mayor sea su probabilidad de ocurrencia y su valor subjetivo. Los efectos agregados del primer grupo de creencias conforman la actitud general.

Luego, el segundo grupo se relaciona con la aprobación o desaprobación del comportamiento por parte de individuos o grupos de referencia importantes. Estas creencias son más fuertes mientras mayor sea la motivación para cumplir con cada uno de los referentes y el grado de aprobación o desaprobación del comportamiento por parte de estos. Los efectos agregados del segundo grupo de creencias conforman la actitud norma subjetiva.

Finalmente, el tercer grupo se relaciona con los recursos y oportunidades, obstáculos o impedimentos percibidos para realizar el comportamiento. Estas creencias son más fuertes mientras existan más factores de control que promuevan o inhiban el comportamiento y mayor sea el poder percibido sobre cada uno de los factores. Los efectos agregados del tercer grupo de creencias conforman la actitud percepción de control sobre el comportamiento.

En efecto, una actitud favorable o desfavorable se forma al evaluar el resultado esperado de la agregación de un grupo de creencias del individuo, formadas a partir de la interpretación de diversos estímulos del entorno. De esta manera, los efectos favorables de la actitud general, la norma subjetiva, la percepción de control sobre el comportamiento y la ponderación relativa de estos determinan la intención de realizar un comportamiento (Ajzen, 1991).

Es importante recalcar que la robustez evidenciada de esta teoría radica en la explicación del proceso cognitivo racional que determina la intención de realizar un comportamiento y no en los factores motivacionales específicos que influyen en esta. Es decir, las creencias específicas que motivan el comportamiento pueden ser conscientes o inconscientes, derivadas del raciocinio o de la emoción, sin que ello afecte el procesamiento de información en el cual los efectos agregados de estas creencias concluyen en una alta probabilidad subjetiva de realizar el comportamiento. El resultado de este proceso se evidencia en la manifestación expresa de la voluntad de realizarlo (Fishbein \& Ajzen, 2010).

Los modelos de intención de emprendimiento tradicionales son adaptaciones o se originan en la teoría del comportamiento planeado, el modelo dominante en la investigación empírica de la intención de emprendimiento (Schlaegel \& Koenig, 2013). 
Cabe resaltar que, desde sus orígenes, los modelos teóricos de intención de emprendimiento (Shapero \& Sokol, 1982) suelen asociar una medida de la intención de emprendimiento a factores motivacionales y rasgos de la personalidad observables y más comunes sin necesariamente implicar causalidad (Ferreira, 2020; Farooq \& Talib, 2019; Kolvereid \& Isaksen, 2006; Davidsson, 1995; Krueger \& Carsrud, 1993; Bird, 1988).

En línea con lo anterior, el implicar causalidad entre factores propuestos y la intención de emprendimiento deben sustentarse en una validación empírica exhaustiva y rigurosa en el ámbito de la psicología a fin de controlar la heterogeneidad de los resultados; no obstante, este sustento no se evidencia en la literatura. Por tanto, a pesar de que algunos autores podrían considerarlo apropiado (Zambrano et al., 2020), el estudio de factores motivacionales o rasgos de personalidad no suele considerarse un estudio de dimensiones o elementos constitutivos de la intención de emprendimiento; por el contrario, la construcción teórica de los modelos de intención se suele sustentar directa o indirectamente sobre la base de las dimensiones de la intención de realizar un comportamiento en la teoría del comportamiento planeado (Acosta-Veliz et al., 2017).

\section{El emprendimiento híbrido}

En la literatura existe terminología alternativa para describir al individuo que mantiene un empleo dependiente y emprende un negocio al mismo tiempo (Demir et al., 2020), como "emprendimiento a tiempo parcial» y moonlighting (empleos múltiples), entre otros (Landgraf, 2015). Esto se debe a una falta de consenso en la concepción, la nomenclatura, los criterios aplicados y la delimitación con conceptos relacionados.

El emprendimiento híbrido puede ser considerado como un punto intermedio en la transición del empleo dependiente al emprendimiento a tiempo completo. Thorgren et al. (2016) definen el proceso de transición del emprendimiento híbrido en dos etapas: la primera, el paso de empleado a emprendedor híbrido; y la segunda, el paso de emprendedor híbrido a emprendedor a tiempo completo. La segunda etapa del emprendimiento híbrido implica la renuncia del empleo para emprender a tiempo completo.

Como se puede apreciar en la figura 1, adaptada de los trabajos de Thorgren et al. (2016) y Wagner (2004), el emprendedor híbrido empieza como emprendedor naciente al tener intención y manifestar esfuerzos de emprendimiento, y tiene la opción de renunciar al empleo para continuar con el emprendimiento a tiempo completo. Esta decisión puede suceder aun cuando el negocio no ha alcanzado ventas suficientes para solventar de manera autónoma costos operativos como la planilla o cuando el negocio ya alcanzó rentabilidad de manera consistente y se considera un emprendimiento joven, el mismo que sigue considerándose emprendimiento, pues no tiene la antigüedad suficiente para concebirse como una empresa establecida (Wagner, 2004). 
Figura 1. Etapas del emprendimiento híbrido en relación a las etapas del proceso emprendedor

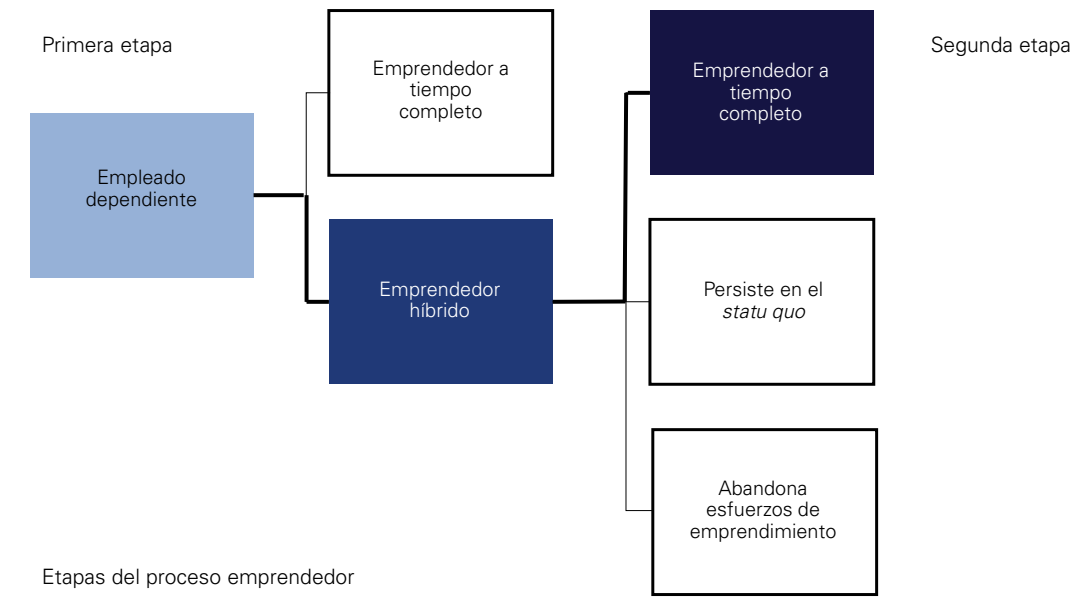

En este sentido, se distingue a los emprendedores híbridos transitorios de los persistentes, pues los primeros tienen intención de renunciar a su empleo dependiente para dedicarse únicamente al emprendimiento en el mediano plazo, mientras que los persistentes desean permanecer realizando ambas actividades indefinidamente aun después de lograr una empresa establecida (Viljamaa \& Varamäki, 2017). Es preciso considerar que el costo de oportunidad de emprender a tiempo completo son las relaciones sociales y beneficios del puesto de trabajo (Cassar, 2006). De no tener la flexibilidad horaria suficiente, el empleado no sería capaz de emprender sin renunciar; pero, si la tiene, tendría la posibilidad de probar su idea de negocio en su tiempo libre, evaluando su desempeño y costo-beneficio para luego tomar una decisión satisfactoria en cuanto a la elección de su ocupación principal (Hellmann, 2007). Según Solesvik (2017), la preferencia por el emprendimiento híbrido se debería a la renuencia a sacrificar el salario y los beneficios no monetarios asociados al empleo, y esa preferencia está asociada a una menor autoconfianza y tolerancia al riesgo que en el caso de los emprendedores tradicionales.

El emprendimiento híbrido transitorio está caracterizado por la intención inicial de empezar el emprendimiento, al mismo tiempo que se mantiene el empleo dependiente, como una opción de inversión real en la cual el individuo gana más información y aprende sobre su propia capacidad de emprendimiento para, luego, decidir si el emprendimiento por sí mismo puede convertirse en la única o mayoritaria fuente de ingresos para él (Folta et al., 2010). Si el individuo confirma su capacidad, procede a renunciar a su empleo para dedicarse principalmente al emprendimiento. En caso no se valide la creencia del emprendedor, este continuará con su negocio como una fuente marginal de ingresos de subsistencia hasta el momento de abandonar sus esfuerzos de emprendimiento (Raffie \& Feng, 2014). Entonces, los factores que influyen en la intención de emprender podrían ser diferentes a los observados en la primera etapa, pues el emprendedor híbrido ajusta sus expectativas, con la ventaja de haber aprendido de su experiencia al poner a prueba su idea de negocio y su capacidad para emprender (Solesvik, 2017; Thorgren et al., 2016). 
Thorgen et al. (2016) indican que se cree que en la segunda etapa existen razones distintas o adicionales a las utilizadas para explicar la elección de emprendimiento en la primera etapa. Asimismo, señalan que la decisión del emprendedor híbrido podría estar basada en qué tan atractiva encuentra la oportunidad de autorrealizarse a través del rol de emprendedor, de forma que al hacerlo deje el empleo dependiente atrás. Entonces, en la transición a la segunda etapa del emprendimiento híbrido se pueden identificar dos componentes o dimensiones principales: una relacionada con la intención de emprender a tiempo completo y otra asociada a la decisión de renunciar al empleo.

Las motivaciones originales entre emprendedores híbridos transitorios y persistentes parecen ser distintas, evidenciando distintos grados de compromiso con el rol emprendedor desde un inicio, compromiso que requiere de cierta valoración e identificación con este rol por parte del individuo (Van der Zwan \& Thurik, 2017). En la literatura, la intención de incursionar y mantenerse en el emprendimiento híbrido ha sido asociada a expectativas o percepción de beneficios monetarios y no monetarios, la pasión por la actividad empresarial, la autoevaluación, la autoeficacia, la inteligencia, la educación, la tolerancia al riesgo y la experiencia en la industria (Luc et al., 2018; Kritskaya et al., 2017; Solesvik, 2017; Block \& Landgraf, 2016).

Por un lado, mientras que los estudios enfocados en la primera etapa del emprendimiento híbrido revelan que los adultos de mediana edad prefieren este tipo de emprendimiento, en la segunda etapa se sugiere que los más jóvenes y los adultos mayores optan por la transición al emprendimiento a tiempo completo. Asimismo, se ha descubierto que las mujeres son más propensas a establecer negocios motivados por la necesidad y a incursionar en el emprendimiento híbrido transitorio (Thorgren et al., 2016).

Por otro lado, respecto de la renuncia al empleo, Klotz et al. (2020) indican que, dentro de las posibles categorías de renuncias, las renuncias asociadas al emprendimiento guardan relación con renuncias voluntarias inevitables por cuestiones de cambio ocupacional o renuncias de la alta gerencia. Los autores comentan que los cambios ocupacionales por parte de emprendedores híbridos están asociados al compromiso organizacional afectivo con el empleo.

Esta investigación propone que la intención de emprendimiento en el emprendedor híbrido puede ser asociada a cuatro factores que también influenciarían la transición de emprendedor híbrido a emprendedor a tiempo completo.

\section{Factores que influencian la transición de emprendedor híbrido a emprendedor a tiempo completo en el modelo conceptual}

Según Marks y Yardley (2004), un tema de investigación surge a partir de un patrón específico encontrado tras un análisis de la información en la que uno está interesado. Acorde a ello, se realizó un análisis cualitativo con información secundaria de la literatura académica para sustentar el desarrollo del modelo conceptual de la intención de emprendimiento en el emprendedor híbrido. Este consistió en un análisis temático de una muestra de treinta y nueve artículos de revistas, principalmente en los cuartiles Q1 y Q2 de sus respectivos campos de estudio, según el ranquin de la Web of Science Journal Citation Reports. Estos artículos fueron obtenidos tras una búsqueda de literatura académica con las palabras clave 
"intención de emprendimiento» y "emprendimiento híbrido» durante el periodo 20002020, puesto que permitía ampliar la muestra con los criterios especificados y evaluar el desarrollo de la literatura. Como resultado, se identificó el uso de ochenta y nueve variables específicas distintas que influenciarían la intención de emprendimiento o la transición al emprendimiento a tiempo completo en diferentes contextos y periodos.

El análisis buscó agrupar estas variables con el objetivo de identificar los principales factores que pueden afectar la intención de emprendimiento e influenciar el proceso de toma de decisiones en el emprendedor híbrido, según la teoría del comportamiento planeado (Ajzen, 1991). Las variables observadas en la literatura responden a creencias que se pueden agrupar y agregar en factores de acuerdo con la naturaleza de las tres dimensiones o actitudes principales que determinan la intención de realizar un comportamiento según dicha teoría, como son la actitud general, la norma subjetiva y la percepción de control sobre el comportamiento. En este sentido, se identificaron cuatro factores diferentes o creencias, como la expectativa de crecimiento empresarial, el capital social emprendedor, el compromiso organizacional con el empleador y la autoeficacia emprendedora, que fueron evidenciados recurrentemente en la literatura del emprendimiento y del emprendimiento híbrido como factores que influencian la decisión de transición al emprendimiento a tiempo completo.

Cabe señalar que los contextos en los cuales los factores propuestos han sido estudiados incluyen al empleado que realiza una transición directa hacia emprendedor a tiempo completo, al empleado que realiza a una transición hacia emprendedor híbrido o al emprendedor híbrido que realiza una transición hacia emprendedor a tiempo completo. A pesar de que los factores señalados han sido estudiados independientemente en la literatura, estos no han sido estudiados en conjunto en el contexto específico del emprendedor híbrido. En este caso, se examinó si los factores propuestos podrían influenciar la intención de emprendimiento que conduce a la transición en la segunda etapa del emprendimiento híbrido; y, para ello, se utilizó el marco de la teoría del comportamiento planeado, que no ha sido aplicada previamente en este contexto.

\subsection{La expectativa de crecimiento empresarial}

La expectativa de los resultados de un comportamiento influye en el proceso de decisión sobre si realizarlo o no. Según Ajzen (1991), una actitud favorable guarda una relación positiva con la intención de realizar un comportamiento, pues manifiesta una disposición general de realizarlo. La actitud general hacia un comportamiento de la teoría del comportamiento planeado se asocia con las expectativas de resultado y también a la magnitud del beneficio o perjuicio que se espere de cada uno de estos. En este sentido, si se espera satisfacer necesidades mediante la realización del comportamiento, la inversión de recursos debería ser menor al beneficio esperado; de lo contrario, no conviene realizar la actividad.

Cabe reiterar que el individuo no siempre es consciente de las creencias o expectativas que motivan su intención y que lo llevan a preferir una opción respecto de otra, y por ello existen elementos no observables que se encuentran implícitos en su evaluación costo-beneficio. Esta limitación es identificada y abordada en la teoría de la utilidad esperada mediante el establecimiento de los axiomas de elección racional que 
cimentan la teoría económica neoclásica y la corriente ortodoxa contemporánea; por ello, son necesarios para suponer la búsqueda de maximización de las utilidades (Von Neumann \& Morgenstern, 1944). En consecuencia, es preciso tener cuidado con asumir a priori que una expectativa de resultado o factor motivacional específico como la expectativa de crecimiento empresarial es una dimensión que guarda una relación causal con la intención de emprendimiento.

Según Botsaris y Vamvaka (2016), la actitud general hacia el comportamiento está formada por la valoración positiva o negativa de cada una de las creencias relacionadas a expectativas de resultado instrumentales, afectivas y de costo de oportunidad. Los autores comentan que las expectativas instrumentales están relacionadas con los beneficios monetarios y profesionales; mientras que las expectativas afectivas están relacionadas con las emociones y sentimientos emanados por el cumplimiento de un rol valorado por el individuo y las expectativas del costo de oportunidad asociado a la decisión de convertirse en un empresario, como podría ser renunciar a la satisfacción proveniente del empleo dependiente y sus beneficios.

En el caso del emprendedor híbrido transitorio observamos expectativas instrumentales, afectivas y de costo de oportunidad. Primero, según Viljamaa y Varamäki (2014) y Viljaama et al. (2014), a diferencia de los emprendedores híbridos persistentes, que estarían a gusto con mantener una utilidad y tamaño de firma pequeños, los transitorios consideran renunciar al empleo para dedicarse al emprendimiento a tiempo completo con la expectativa de conseguir una utilidad superior a la que gozan gracias al crecimiento de su negocio. En este sentido, según Brown y Farshid (2017), para obtener beneficios superiores, los esfuerzos necesarios para el crecimiento de las ventas suponen una mayor participación del emprendedor en su negocio y una mayor inversión de recursos que podrían dificultar mantener el empleo dependiente al mismo tiempo. Segundo, Van der Zwan y Thurik (2017) comentan que, adicionalmente, existen expectativas afectivas mediante el compromiso con el rol de emprendedor manifestado en los esfuerzos para lograr el objetivo de una empresa establecida. Tercero, evidenciamos la expectativa de un menor costo de oportunidad, pues si los beneficios futuros esperados son superiores a los obtenidos en el empleo dependiente, entonces el emprendedor híbrido dará prioridad al negocio por encima del empleo (Block \& Landgraf, 2016).

El crecimiento empresarial depende de las dinámicas del mercado y suele medirse en términos de crecimiento porcentual de ventas, de número de empleados o de un promedio de ambos durante un periodo específico (Levie \& Autio, 2013). Sin embargo, aún con condiciones favorables en el mercado, si bien el crecimiento del negocio se asocia a recompensas financieras o a un mayor grado de independencia, este no siempre resulta deseable por la posible reducción del bienestar en los empleados, la pérdida de control en la supervisión y el aumento de la carga de trabajo que generaría (Davidsson, 1989). Asimismo, es preciso reconocer que la expectativa de crecimiento empresarial suele ser definida por el emprendedor aun cuando las condiciones del mercado no sean favorables, y que se podría medir a través de las metas u objetivos de porcentaje de crecimiento de ventas o de número empleados que este espera conseguir en los próximos cinco años (Delmar \& Wiklund, 2008). 
Literatura reciente sobre intención y transición al emprendimiento a tiempo completo ha evidenciado que ambos conceptos están asociados positivamente a la actitud general, representada en las expectativas de alto crecimiento que convierten el emprendimiento en una opción de carrera factible (Marshall \& Gigliotti, 2018; Viljamaa \& Varamäki, 2017; Viljamaa et al., 2014b), actitud representada también en la búsqueda del éxito financiero (Block \& Landgraf, 2016) y la propiedad sobre el negocio (Douglas \& Fitzsimmons, 2013). En esencia, estas variables estarían conceptualmente asociadas a los beneficios que generaría invertir en el crecimiento de un negocio exitoso y, por tanto, a la expectativa de crecimiento empresarial del emprendedor.

En conclusión, la evaluación positiva de un análisis costo-beneficio genera la expectativa de crecimiento empresarial que motiva los esfuerzos que supone este crecimiento (Levie \& Autio, 2013), como renunciar al empleo para dedicarse al emprendimiento a tiempo completo al considerarse esta opción de carrera deseable en el caso de los emprendedores híbridos (Marshall \& Gigliotti, 2018; Shapero \& Sokol, 1982). Según lo anterior, en la presente investigación se considera la siguiente proposición: P1 La expectativa de crecimiento empresarial está asociada positivamente a la intención de emprendimiento a tiempo completo en emprendedores híbridos.

\subsection{El capital social emprendedor}

A diferencia del capital físico que se puede acumular, el capital social es intangible y difícil de identificar y cuantificar, por lo que se suele estudiar por las características observables que manifiesta, como interacciones, la formación de agrupaciones, las creencias, los valores culturales y las normas sociales explícitas e implícitas que regulan el comportamiento; y, a un nivel más personal, las características sociables de la persona, que la hacen más o menos propensa a recurrir a su red de contactos (Anderson et al., 2007). Las redes de contactos son grupos de individuos o agentes que se involucran en actividades para obtener recursos (Bowey \& Easton, 2007).

Se suele pensar que el capital social emprendedor está constituido por los recursos implícitos o potenciales que favorecen el emprendimiento y se derivan de la red de contactos (Anderson et al., 2007). Este tipo de capital es importante porque permite al individuo percibir un mayor acceso a recursos, ya sea mediante intercambio de bienes, servicios o conocimiento (Westlund \& Bolton, 2003).

Entonces, en línea con lo señalado por Bowey y Easton (2007), el capital social puede entenderse como la credibilidad en el compromiso de un comportamiento cooperativo, está asociado a la calidad y cercanía de las relaciones sociales, y se encuentra implícito en la interacción social que se produce al realizar una actividad. Esta interacción social se manifiesta al prestar, encomendar, negociar e intercambiar recursos económicos y sociales en búsqueda de beneficio mutuo, por ejemplo, intercambiando favores, comentarios o rumores.

La credibilidad dentro de una red de contactos se asocia indirectamente a la posible obtención de recursos y, por ello, el capital social de un empleado es valioso para su organización en cuanto al capital humano por su capacidad de generar márgenes de ganancia que se ven reflejados en su salario (Campbell et al., 2017). Así, el empleado 
puede usar su red de contactos de negocios, profesionales y personales para conseguir información y proveer soluciones empresariales (Fernandez-Pérez et al., 2015; Campopiano et al., 2014).

La asesoría que podría recibir el individuo a través de su red de contactos incrementa su probabilidad subjetiva de éxito en el emprendimiento (Luc et al., 2018; Hui-Chen et al., 2014); por este motivo, las organizaciones procuran retener talento, compensando en mayor medida a los empleados más competitivos, de tal manera que el valor esperado de los beneficios de buscar otro empleo o emprender sean menores a los beneficios recibidos por la organización (Campbell et al., 2017; Liñán et al., 2011).

Este último punto estaría directamente relacionado con las actitudes de norma subjetiva y de percepción de control del comportamiento de la teoría del comportamiento planeado, puesto que los efectos ponderados de las creencias de percepciones por parte de otros individuos o grupos importantes para el individuo afectarían su percepción de la probabilidad de éxito del negocio, el deseo y factibilidad percibida del negocio, y, por tanto, la intención de emprendimiento. El capital social emprendedor es un factor latente que se podría medir a través del puntaje promedio de las escalas de Likert 1-7 que calculen la familiaridad con el apoyo estatal al emprendimiento; la familiaridad con capacitación en temas relacionados al emprendimiento, al financiamiento, al apoyo técnico y al grado de vinculación con centros de negocios; la valoración del rol emprendedor por parte de familia, amigos y colegas; el grado de creencia de la contribución del emprendedor a la economía; $y$, finalmente, el grado de creencia de una cultura desfavorable para el emprendimiento en el país, en relación al porcentaje de población que considera que el emprendedor se aprovecha u explota a otras personas en beneficio propio (Vukovic \& Kedmenec, 2017).

En conclusión, el capital social emprendedor implica relaciones del emprendedor híbrido con otros individuos o instituciones que favorecen el emprendimiento dentro de su red de contactos (Vukovic \& Kedmenec, 2017). La valoración del rol emprendedor por parte de su entorno le permite obtener beneficios como la satisfacción de la necesidad psicológica básica de sentido de pertenencia (Ryan \& Deci, 1980) o recursos para el emprendimiento que generen una ventaja competitiva (Barney, 1991). Estos beneficios esperados estarían asociados con una mayor intención de emprendimiento. Según lo anterior, en esta investigación se considera la siguiente proposición: P2 - El capital social emprendedor está asociado positivamente a la intención de emprendimiento a tiempo completo en emprendedores híbridos.

\subsection{El compromiso organizacional con el empleador}

La intención de renunciar no se estudia directamente para predecir las renuncias por sus resultados inconsistentes, por lo que se suele utilizar el compromiso organizacional para ello (Boshoff \& Mels, 2000; Hunt \& Morgan, 1994).

Las diferencias conceptuales entre el compromiso y la motivación radican en que los compromisos involucran decisiones de largo plazo, mientras que la motivación es un concepto más general asociado a todo tipo de decisiones y, generalmente, se relaciona con el corto plazo en la realización de actividades sencillas. Esto quiere decir que el compromiso está implícito en la motivación, de tal manera que contribuye a las razones que conllevan a 
la intención de realizar un determinado comportamiento, como renunciar al empleo (Meyer et al., 2004).

Según Meyer y Allen (1991), el compromiso organizacional es el lazo afectivo construido a partir de la identificación con los valores de la organización, el deseo de afiliación y de cumplir sus normas; también está asociado a la obligación moral de corresponder al empleador por los beneficios obtenidos en el empleo. Asimismo, es un componente temporalmente estable que se construye con el tiempo y es resultado de la acumulación continua de otros factores, como la satisfacción laboral o la implicación en el trabajo.

En línea con lo anterior, el compromiso organizacional es también una manifestación del sentido de pertenencia generado por las buenas relaciones entre el empleado y sus jefes y compañeros, el gusto por las tareas realizadas, los valores compartidos, y la satisfacción con las condiciones salariales y los beneficios que retienen a un empleado en la organización; por ello, ha sido ampliamente estudiado como predictor de renuncia (Meyer et al., 2004; Tett \& Meyer, 1993; Ryan \& Deci, 1980). Por tanto, el compromiso organizacional se considera un buen predictor de las intenciones de permanecer en la empresa (Solinger et al., 2008).

Estudiando con más profundidad la relación del empleado con su empleador, literatura reciente sobre intención y transición al emprendimiento a tiempo completo ha evidenciado que ambos conceptos están asociados negativamente a la satisfacción laboral en el empleo (Sun et al., 2019; Marshall, 2017; Guerra \& Patuelli, 2016), incluyendo la satisfacción asociada a recompensas (Mourmant et al., 2009), cambios respecto a condiciones laborales y salario (Klotz et al., 2020; Failla et al., 2017; Elfenbein et al., 2010). Si bien aquellos que ganan un mayor salario y obtienen más beneficios laborales son menos propensos al emprendimiento, estos individuos son los que suelen renunciar al empleo para emprender a tiempo completo (Guerra \& Patuelli, 2016; Douglas \& Fitzsimmons, 2013; Andersson \& Wadensjö, 2011).

El emprendimiento híbrido ha sido considerado una condición inestable por las exigencias del empleo y el creciente número de exigencias de crecimiento del negocio para sobrevivir en un entorno altamente competitivo, dado que el eminente conflicto entre ambos roles lleva al emprendedor a inclinarse por alguno de estos y, eventualmente, abandonar o renunciar al otro de manera irremediable (Marshall, 2017). Una de las razones es que el emprendedor híbrido desarrolla gradualmente una identidad de emprendedor $y$, de tener éxito, la llega a valorar más que su identidad como empleado manifiesta en su compromiso organizacional afectivo (Klotz et al., 2020). Este cambio de identidad se asocia a valores preciados por el individuo, como la autonomía o independencia, que podrían no estar siendo satisfechos en el empleo dependiente (Douglas \& Fitzsimmons, 2013; Mourmant et al., 2009).

Dedicarse al emprendimiento a tiempo completo en el contexto de la segunda etapa del emprendimiento híbrido involucra renunciar al empleo dependiente y, por tanto, el emprendedor híbrido transitorio debería tener la intención de renunciar al empleo en un mediano plazo (Thorgren et al., 2016). No obstante, el compromiso organizacional con el empleador podría influenciar en la decisión de no renunciar por los beneficios monetarios y no monetarios que genera al individuo (Hausknecht et al., 2009), por lo que el empleado tendría que pasar por un proceso de desvinculación con el entorno laboral para renunciar. 
Este último punto relacionaría el compromiso organizacional con el empleador directamente a la actitud de norma subjetiva de la teoría del comportamiento planeado, puesto que los efectos ponderados de las creencias de percepciones por parte de otros individuos o grupos importantes para el individuo, como familia, amigos y colegas, afectarían el deseo de emprender y, por tanto, la intención de emprendimiento. El compromiso organizacional se suele medir aplicando el cuestionario Organizational Commitment Questionnaire de quince ítems elaborado por Mowday et al. (1979). Los autores Bozemann y Perrewé (2001), por su parte, sugieren utilizar nueve ítems de los originales, dado que estos han demostrado un mayor grado de validez en la predicción de la intención de permanecer en la empresa en donde el individuo está empleado.

En conclusión, el compromiso organizacional con el empleador, que implica una buena relación entre este, el emprendedor híbrido y otros individuos de su entorno laboral (Hunt \& Morgan, 1994), permite obtener beneficios como la satisfacción de la necesidad psicológica básica de sentido de pertenencia (Ryan \& Deci, 1980) o mayor satisfacción laboral asociada al salario, beneficios sociales, funciones, tareas y colegas (Hausknecht et al., 2009). Entonces, el compromiso organizacional con el empleador estaría asociado a una menor intención de renunciar para dedicarse al emprendimiento a tiempo completo (Klotz et al., 2020). Según lo anterior, en esta investigación se considera la siguiente proposición: P3 - El compromiso organizacional con el empleador está asociado negativamente a la intención de emprendimiento a tiempo completo en emprendedores híbridos.

\subsection{La autoeficacia emprendedora}

Según Ajzen (1991), la percepción de control del comportamiento ha sido inspirada en el constructo de autoeficacia propuesto por Bandura (1986) y está formada por la evaluación de las creencias relacionadas con percepciones de factibilidad para lograr un buen desempeño en el desarrollo de una actividad, lo que implica los recursos, las oportunidades y las amenazas percibidas por el individuo que podrían facilitar o dificultar el llevar a cabo el comportamiento. Solesvik (2017) comenta que la autoeficacia es uno de los predictores más consistentes de la intención de emprendimiento.

Bandura (1977) define la autoeficacia como la expectativa de la propia capacidad para desempeñar un comportamiento con éxito y marca la diferencia de este constructo con las expectativas de resultado, porque estas son una estimación personal de los efectos de realizar una determinada acción bajo el supuesto de que esta se realiza exitosamente. Si el individuo tiene serias dudas sobre su capacidad para realizar un comportamiento, entonces no lo realizará a pesar de los beneficios asociados. Asimismo, el individuo tiene diferentes fuentes de información para evaluar su propia capacidad, como el desempeño en comportamientos exitosos en el pasado, la experiencia vicaria o aprendida a partir de terceros, y la persuasión verbal como autosugestión y excitación emocional por la vinculación afectiva con el comportamiento a realizar.

Según Chen et al. (1998), la autoeficacia emprendedora es la fuerza de las creencias asociadas a la propia capacidad para desempeñarse satisfactoriamente en el rol y las tareas de un emprendedor. En este sentido, Zhao et al. (2005) sugieren que la autoeficacia emprendedora media los efectos del aprendizaje o la educación formal, la experiencia en 
emprendimiento y la propensión a tomar riesgos en la intención de emprendimiento. La autoeficacia resulta un buen predictor de la intención de emprendimiento porque puede mediar el efecto agregado de otros constructos asociados a la intención de emprendimiento, ya que modela las creencias y expectativas de desempeño del individuo y, por ello, su nivel de confianza en su propia capacidad de emprender.

Algunos estudios asocian la autoeficacia emprendedora a una mayor percepción de factibilidad del emprendimiento. Por un lado, Neneh (2020) comenta que la autoeficacia emprendedora está positivamente asociada a la intención de emprendimiento híbrido y a tiempo completo, dependiendo del contexto en el que se analice. En línea con lo anterior, sugiere que la propia percepción de empleabilidad está asociada negativamente a la transición al emprendimiento a tiempo completo. En ese sentido, aquellos que se perciben como menos capaces para conseguir empleo respecto de sus habilidades para emprender, prefieren el emprendimiento a tiempo completo; mientras que los que se perciben como más capaces para conseguir empleo respecto de sus habilidades para emprender, prefieren el emprendimiento híbrido.

Este último punto relacionaría la autoeficacia emprendedora directamente a la actitud de percepción de control del comportamiento de la teoría del comportamiento planeado, puesto que los efectos ponderados de las creencias en el control que se tiene sobre los resultados del negocio en términos de la propia capacidad de emprender con éxito afectarían la factibilidad percibida del negocio y, por tanto, la intención de emprendimiento. La autoeficacia emprendedora es un factor latente que se podría medir mediante el puntaje promedio de las escalas de Likert 1-5 relacionadas al grado de confianza en la propia capacidad de identificación de nuevas oportunidades de negocio, de creación de nuevos productos, de pensamiento creativo y de comercialización de una idea o nuevo descubrimiento (Zhao et al., 2005).

En conclusión, la autoeficacia emprendedora implica la confianza del emprendedor híbrido en sus capacidades para establecer una empresa con éxito (Raffie \& Feng, 2014). Al ser validada por el entorno, le permite obtener beneficios como la satisfacción de la necesidad psicológica básica de sentido de competencia (Ryan \& Deci, 1980) y la necesidad de logro (McClelland, 1961). Estos beneficios esperados generan mayor disposición a realizar esfuerzos de emprendimiento, pues es una opción factible (Shapero \& Sokol, 1982). Así, la autoeficacia emprendedora se asocia a una mayor intención de emprendimiento a tiempo completo. Según lo anterior, en esta investigación se considera la siguiente proposición: P4 - La autoeficacia emprendedora está asociada positivamente a la intención de emprendimiento a tiempo completo en emprendedores híbridos.

En la figura 2, se presenta un esquema resumen de las relaciones teóricas antes mencionadas entre los factores propuestos y las dimensiones de la intención para el caso del emprendimiento a tiempo completo en el contexto del emprendedor híbrido. Como se puede apreciar, la expectativa de crecimiento empresarial estaría asociada a la actitud general, el compromiso organizacional con el empleador estaría asociado a la actitud de norma subjetiva, la autoeficacia emprendedora estaría asociada a la actitud de percepción de control del comportamiento y, finalmente, el capital social emprendedor estaría asociado a ambas actitudes de norma subjetiva y percepción de control sobre el comportamiento. 
Teoría del comportamiento planeado

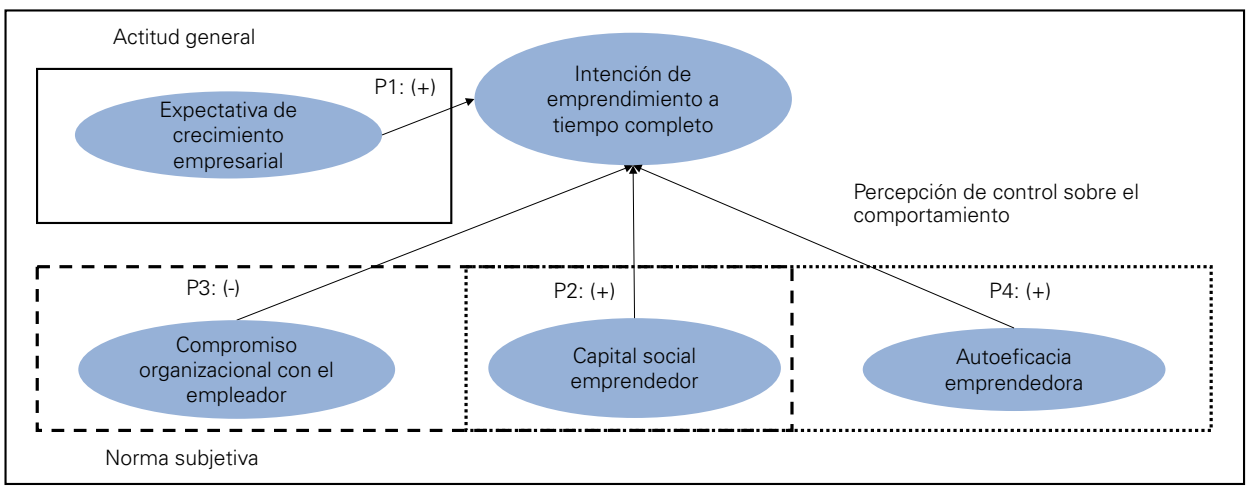

Fuente: elaboración propia.

\section{Discusión}

El emprendimiento híbrido es un fenómeno que ha cobrado relevancia en la última década y su literatura se encuentra en constante desarrollo. En base a la teoría del comportamiento planeado, esta investigación examina la segunda etapa del emprendimiento híbrido, en la cual el emprendedor híbrido toma una decisión entre continuar realizando ambas actividades, trabajar en un empleo dependiente y en un negocio propio, o renunciar a alguna de las dos.

Esta investigación brinda la oportunidad de profundizar en el estudio de los factores que influenciarían la intención de transición al emprendimiento a tiempo completo en la segunda etapa del emprendimiento híbrido. Si bien hay estudios relacionados con la autoeficacia, el efecto del capital social emprendedor, el compromiso organizacional y las expectativas de crecimiento empresarial en la literatura de intención de emprendimiento, estos factores no han sido estudiados en conjunto en el contexto del emprendedor híbrido. Se espera que los resultados de la futura aplicación del modelo planteado de los factores que influyen en la intención de emprender a tiempo completo en emprendedores híbridos evidencien que un incremento en las expectativas de crecimiento empresarial, el capital social emprendedor y la autoeficacia emprendedora tendría una relación positiva con la intención de emprendimiento en emprendedores híbridos. Asimismo, se espera que también evidencie que un incremento en el compromiso organizacional con el empleador tendría una relación negativa respecto de la intención de emprendimiento en este grupo.

Mientras que Thorgren et al. (2016) definen la segunda etapa del emprendimiento híbrido de manera general como aquella en la que un emprendedor híbrido toma la decisión de renunciar a su empleo para continuar con su emprendimiento, esta investigación ahonda en el proceso de toma de decisiones que precede a la transición. Para ello, identificó creencias específicas estrechamente relacionadas que determinarían la intención de emprendimiento a tiempo completo, en el marco de los tipos de creencias que conforman las actitudes genéricas de actitud general, norma subjetiva y percepción de control del comportamiento que, teóricamente, determinan la intención de realizar un comportamiento de acuerdo con la teoría del comportamiento planeado. En línea con lo anterior, lo propuesto 
es consecuente con el uso dominante de esta teoría para determinar empíricamente la intención de emprendimiento, como señalan Schlaegel y Koenig (2013) en base a un metaanálisis; y con el uso previo de esta teoría para evaluar factores que influenciarían la intención de emprendimiento en la primera etapa del emprendimiento híbrido, como en el caso de Farooq y Talib (2019). Al respecto, no se encontró evidencia de estudios similares en la segunda etapa del emprendimiento híbrido.

La intención de emprendimiento no sería la misma entre ambas etapas del emprendimiento híbrido debido a la experiencia de aprendizaje entre ellas (Ferreira, 2020; Raffie \& Feng, 2014; Folta et al., 2010), lo que podría explicarse en determinantes distintos o adicionales a los iniciales que permitieron la transición de empleado a emprendedor híbrido, como la necesidad de un ingreso adicional o la pasión por un pasatiempo evidenciados en el emprendimiento híbrido persistente (Viljamaa \& Varamäki, 2017; Thorgren et al., 2014). En este sentido, la expectativa o ambición de beneficios monetarios superiores a los obtenidos en el estado actual (Block \& Landgraf, 2016), la percepción de una mayor cantidad de recursos para invertir debido al apoyo moral y económico de una mayor red de contactos de negocios (Vukovic \& Kedmenec, 2017), y la percepción de una mayor capacidad individual para el emprendimiento (Ferreira, 2020; Solesvik, 2017) estarían asociadas a una mayor intención de emprendimiento; $y$, en contraste, un mayor nivel de compromiso organizacional afectivo con el empleador retendría al emprendedor híbrido en su empleo dependiente, reduciendo su intención de emprendimiento (Klotz et al., 2020). No obstante, aún no se han analizado estos elementos en conjunto para efectos de determinar la intención de emprendimiento en la segunda etapa del emprendimiento híbrido.

Al examinar la intención de emprendimiento en la segunda etapa del emprendimiento híbrido, esta investigación hace alusión a las sugerencias de mayor investigación por parte de Ferreira (2020), Solesvik (2017), Thorgren et al. (2016) y otros investigadores con el objetivo de expandir el campo de la literatura sobre el emprendimiento híbrido (Luc et al., 2018; Viljamaa \& Varamäki, 2017; Block \& Landgraf, 2016). El aporte principal de esta investigación conceptual radica en su enfoque multidisciplinario, que utiliza la aplicación de una teoría existente en un contexto diferente y, por lo tanto, amplía el alcance del pensamiento común respecto de la materia de análisis (Gilson \& Goldberg, 2015) y ofrece un modelo conceptual con proposiciones debidamente sustentadas para su evaluación empírica posterior (Weick, 1989).

\section{Implicancias prácticas}

El emprendedor híbrido transitorio y su decisión de convertirse en emprendedor a tiempo completo han cobrado relevancia debido a la gran cantidad de emprendedores nacientes que se registra en países como Suecia, Estados Unidos, Reino Unido, Alemania, Noruega y México; y a sus efectos sobre el crecimiento económico (Ferreira, 2020; Sun et al., 2019; Luc et al., 2018; Kritskaya et al., 2017; Viljamaa \& Varamäki, 2017; Block \& Landgraf, 2016).

Asimismo, se ha evidenciado que los negocios de emprendedores híbridos tienen mayor tasa de sobrevivencia empresarial, asociada a una mayor generación de empleo en la economía (Raffie \& Feng, 2014). Además, estos individuos suelen ser más receptivos a 
los programas de fomento al emprendimiento, por lo que enfocar políticas a este segmento deriva en una mayor efectividad de dichas políticas públicas (Schulz et al., 2016). Por tanto, el investigar los factores que motivarían que un emprendedor híbrido decida dedicarse a su emprendimiento a tiempo completo sería beneficioso para los grupos de interés del entorno en el que se desarrolla.

En este sentido, esta investigación aportaría a llenar un vacío en la creciente literatura académica de emprendimiento híbrido al ofrecer un modelo conceptual aplicado a un contexto novedoso y plantear proposiciones para su posterior validación empírica, considerando que aún no se ha aplicado la teoría del comportamiento planeado para determinar la intención de emprendimiento en el contexto de la segunda etapa del emprendimiento híbrido ni se han evaluado en conjunto los determinantes propuestos sobre la intención de emprendimiento en este contexto.

\section{Limitaciones de la investigación}

Esta investigación no carece de limitaciones, algunas de las cuales se presentan a continuación. En primer lugar, esta investigación no busca modelar la conducta, sino la intención actual, dado que existen elementos adicionales que podrían impulsar una conducta futura diferente a la esperada. En este caso, se buscan creencias asociadas a factores actitudinales robustos en la determinación de la intención de emprendimiento a tiempo completo en el emprendedor híbrido y se requiere de un análisis adicional para predecir el comportamiento.

En segundo lugar, como se trata de un estudio conceptual, no se puede asumir que los factores propuestos ofrecen una lista completa y exhaustiva de todas las variables que podrían influir en la transición de emprendedor híbrido a emprendedor a tiempo completo.

En tercer lugar, el modelo conceptual es diseñado de manera genérica, como es común en la elaboración de modelos teóricos, por lo que no se circunscribe a un entorno específico; por lo tanto, no controla los efectos de todos los factores personales de un individuo ni de los factores del entorno macroeconómico. En consecuencia, en ciertos contextos geográficos, los efectos de algunos de los factores identificados podrían ser matizados; por ejemplo, algunos patrones culturales o factores sociodemográficos podrían tener influencia en la toma de decisiones.

En cuarto lugar, a medida que la literatura de emprendimiento híbrido se desarrolla, es posible que el modelo conceptual propuesto evolucione para incorporar los nuevos descubrimientos.

\section{Conclusiones y recomendaciones para futuras investigaciones}

A pesar de que la existencia de individuos que realizan actividades relacionadas a un empleo dependiente y a un emprendimiento en simultáneo no es reciente, aún es un reto para las conceptualizaciones tradicionales de emprendimiento, en las que se considera que el emprendedor se dedica únicamente a realizar esfuerzos para establecer una nueva empresa; y, por ello, podrían existir definiciones alternativas del mismo fenómeno como emprendimiento híbrido, emprendimiento a tiempo parcial o considerarlo como una segunda 
ocupación, sin tomar en cuenta la variedad de formas en las que el emprendimiento se manifiesta. Debido a ello, aún se sigue evaluando la prevalencia de este fenómeno a nivel global.

Esta investigación ahonda en el proceso de toma de decisiones del emprendedor híbrido en la segunda etapa, en la cual decide renunciar al empleo para dedicarse por completo al emprendimiento. Específicamente, examina la intención de emprendimiento a tiempo completo que se manifiesta de manera previa a la transición, proponiendo cuatro factores o creencias que podrían determinar dicha intención. La contribución de cada creencia sobre la intención está sustentada en la naturaleza de las tres principales actitudes que determinan la intención de realizar un comportamiento, según la teoría del comportamiento planeado. A partir de esta teoría, se desarrolló un modelo conceptual y también algunas proposiciones para guiar futuras investigaciones empíricas asociadas a este tema, con lo que se busca promover el desarrollo de la literatura de emprendimiento híbrido en la segunda etapa. A continuación, se presentan algunas recomendaciones para futuras investigaciones.

Primero, se sugiere evaluar empíricamente el modelo propuesto, lo que podría derivar en factores adicionales que influencien la intención de emprendimiento en la segunda etapa del emprendimiento híbrido. Para efectos de la evaluación empírica, se sugiere utilizar variables de control con el objetivo de separar sus efectos de aquellos factores que se buscan validar mediante pruebas de hipótesis. Algunas de las variables de control usualmente utilizadas en la literatura son las de género (Folta et al., 2010), edad (Thorgren et al., 2016), nivel educativo (Block \& Landgraf, 2016) y ámbito geográfico (Liñan \& Santos, 2007), entre otras. Por otro lado, existen múltiples definiciones de emprendimiento a tiempo completo o a tiempo parcial en la literatura; así, algunos autores asocian la dedicación a tiempo completo con la ocupación principal declarada y la ocupación de donde proviene la mayor proporción de ingresos, o utilizan como criterio la jornada laboral vigente de su contexto geográfico específico, por lo cual también se sugiere acotar dicho concepto para efectos de la evaluación empírica (Landgraf, 2015).

Segundo, siguiendo las recomendaciones de Ferreira (2020), las investigaciones relacionadas con motivaciones individuales e influencias normativas brindarían un mayor entendimiento sobre los factores que influencian la intención en la segunda etapa del emprendimiento híbrido. En línea con lo anterior, siguiendo la definición de segunda etapa establecida por Thorgren et al. (2016), las investigaciones en la literatura de emprendimiento híbrido no suelen considerar aspectos relacionados a la intención de renunciar al empleo, en cuya ausencia no se daría la transición esperada; por ello, se sugiere considerar algún factor adicional relacionado a la renuncia o permanencia en el empleo.

Tercero, los próximos estudios que decidan focalizarse en un área geográfica en particular deberían tener en cuenta factores del entorno y, a partir de ello, identificar efectos en base a factores culturales, sociales, políticos o económicos específicos, considerando también el uso de análisis comparativos entre distintas poblaciones.

Finalmente, de acuerdo con Bögenhold (2019), deben tomarse en cuenta las dificultades de obtención de datos en el diseño de investigación, pues podría no disponerse de una base de datos asociada al emprendimiento híbrido. En este sentido, se sugiere continuar validando empíricamente modelos ya existentes en la literatura en desarrollo, fomentando así la visibilidad del emprendimiento híbrido en la literatura. 


\section{bibliografía}

\section{Acosta-Veliz, M., Villacís-Aveiga,} W., \& Jiménez-Cercado, M.

2017 Factores que conforman la intención emprendedora de estudiantes de la Universidad de Guayaquil. Dominio de las Ciencias, 3(3), 335-346. https://dialnet. unirioja.es/descarga/articulo/6244045.pdf

\section{Ajzen, I.}

The theory of planned behavior.

Organization Behavior and Human Decision Processes, 50(2), 179-211. https://doi. org/10.1016/0749-5978(91)90020-T

\section{Anderson, A., Park, J., \& Jack, S.}

Entrepreneurial Social Capital:

Conceptualizing Social Capital in New

High-tech Firms. International Small

Business Journal, 25(3). https://doi.

org/10.1177/0266242607076526

\section{Andersson, P., \& Wadensjö, E.}

The best and the brightest or the least successful? Self-employment entry among male wage-earners in Sweden. Small Business Economics, (40), 155-172 http://dx.doi.org/10.1007/s11187-011-9365-0

\section{Atkinson, J.}

An introduction to motivation. Princeton,

Nueva Jersey: D. Van Nostrand.

\section{Bandura, A.}

Self-efficacy: Toward a unifying theory of behavioral change. Psychological Review, 84(2), 191-215. https://psycnet.apa.org/ doi/10.1037/0033-295X.84.2.191

\section{Bandura, A.}

1991

\section{Barney, J.}

Firm resources and sustained competitive advantage. Journal of management, 17(1). https://doi. org/10.1177/014920639101700108

\section{Bird, B.}

Implementing Entrepreneurial Ideas:

The Case for Intention. Academy of Management Review, 13(3), 442-453. https://doi.org/10.5465/amr.1988.4306970

\section{Block, J., \& Landgraf, A.}

Transition from part-time entrepreneurship to full-time entrepreneurship: the role of financial and non-financial motives. International Entrepreneurship And Management Journal, (12), 259-282. https://doi.org/10.1007/s11365-014-0331-6

\section{Bögenhold, D.}

2018 From Hybrid Entrepreneurs to Entrepreneurial Billionaires: Observations on the Socioeconomic Heterogeneity of Self-employment. American Behavioral Scientist, 63(2). https://doi. org/10.1177\%2F0002764218794231

\section{Bögenhold, D.}

2019 Are hybrids the new normal? A labour market perspective on hybrid selfemployment. International Review of Entrepreneurship, 17(4), 429-447.

\section{Boshoff, C., \& Mels, G.}

The Impact of Multiple Commitments on Intentions to Resign: an Empirical Assessment. British Journal of Management, 11(3), 255-272. https://doi. org/10.1111/1467-8551.00165. 


\section{bibliografía}

Botsaris, C., \& Vamvaka, V.

2016

Attitude Toward Entrepreneurship:

Structure, Prediction from Behavioral

Beliefs, and Relation to Entrepreneuria

Intention. Journal Of The Knowledge

Economy, (7), 433-460. https://doi

org/10.1007/s 13132-014-0227-2

Bowey, J., \& Easton, G.

2007

Entrepreneurial Social Capital Unplugged:

An Activity-based Analysis. International

Small Business Journal: Researching

Entrepreneurship, 25(3). https://doi.

org/10.1177/0266242607076528

\section{Bozemann, D., \& Perrewé, P.}

The Effect of Item Content Overlap on

Organizational Commitment QuestionnaireTurnover Cognitions Relationships. Journal of Applied Psychology, 86(1), 161-173.

\section{Brown, T., \& Farshid, M.}

To grow or not to grow, that is the question. Entreprendre \& Innover, 3(34), 29-37. https://doi.org/10.3917/ entin.034.0029

Burke, A. E., FitzRoy, F. R., \& Nolan, M. A.

What makes a die-hard entrepreneur? Beyond the 'employee or entrepreneur' dichotomy. Small Business Economics, (31). https://link.springer.com/ article/10.1007/s11187-007-9086-6

Campbell, B. A., Kryscynski, D., \& Olson, D. M.

Employee Entrepreneurship Research: A Labor Market Frictions Approach. Strategic Entrepreneurship Journal, 11(3), 344-356. https://doi.org/10.1002/sej.1264

Campopiano, G., Minola, T., \& Sainaghi, R.

2014

Family Resources and Entrepreneurial Intention in Hospitality and Tourism Industry. ICSB World Conference Proceedings.

\section{Cassar, G.}

Entrepreneur opportunity costs and intended venture growth. Journal of Business Venturing, 21(5), 610-632. https:// doi.org/10.1016/j.jbusvent.2005.02.011

\section{Chen, G., \& Crick, A}

Does entrepreneurial self-efficacy distinguish entrepreneurs from managers? Journal of Business Venturing, 13(4), 295-316. https://doi.org/10.1016/S08839026(97)00029-3

\section{Davidsson, $\mathbf{P}$.}

Entrepreneurship... and after? A study of Growth Willingness in Small Firms. Journal of Business Venturing, 4(3), 211-226. https://doi.org/10.1016/08839026(89)90022-0

\section{Davidsson, $\mathbf{P}$.}

Determinants of Entrepreneurial Intentions. RENT IX Workshop, 11, pp. 23-24. https://eprints.qut.edu.au/2076/

\section{Delmar, F., \& Wiklund, J.}

The Effect of Small Business Managers' Growth Motivation on Firm Growth: A Longitudinal Study. Entrepreneurship Theory and Practice, 32(3), 437-457. https://doi.org/10.1111\%2Fj.15406520.2008.00235.x 


\section{bibliografía}

Demir, C., Werner, A., Kraus, S.,

\section{\& Jones, $\mathrm{P}$.}

2020

Hybrid Entrepreneurship: A Systematic

Literature Review. Journal of Small

Business \& Entrepreneurship. http://dx.doi.

org/10.1080/08276331.2020.1764738

\section{Douglas, E., \& Fitzsimmons, J.}

Intrapreneurial intentions versus

entrepreneurial intentions: distinct

constructs with different antecedents.

Small Business Economics, (41), 115-132.

https://doi.org/10.1007/s11187-012-9419-y

\section{Elfenbein, H., \& Zenger.}

2010 The Small Firm Effect and the

Entrepreneurial Spawning of Scientists

and Engineers. Management Science,

56(4), 659-681. https://doi.org/10.1287/

mnsc. 1090.1130

Failla, V., Melillo, F., \&

\section{Reichstein, T.}

Entrepreneurship and employment stability - Job matching, labour market value, and personal commitment. Journal of Bussines Venturing, 32(2), 162-177. https://doi. org/10.1016/j.jbusvent.2017.01.002

\section{Farooq, M., \& Talib, N.}

Hybrid Entrepreneurial Intention: A

Comparative Study of Public and Private

Sector Employees. Journal of Research in Psychology, 1(1), 21-26. https://doi. org/10.31580/jrp.v1i1.522

\section{Fernández-Pérez, V., Alonso-}

Galicia, P. E., Rodríguez-Ariza, L., \& Fuentes-Fuentes, M. del M.

A bridge to entrepreneurship for academics? European Management

Journal, 33(1), 37-47. https://doi. org/10.1016/j.emj.2014.07.003

\section{Ferreira, C.}

2020

Experiential learning theory and hybrid entrepreneurship: factors influencing the transition to full-time entrepreneurship. International Journal of Entrepreneurial Behavior \& Research, 26(8). https://doi. org/10.1108/IJEBR-12-2019-0668

\section{Fishbein, M., \& Ajzen, I.}

1975 Belief, Attitude, Intention, and Behavior: An Introduction to Theory and Research. Reading: Addison-Wesley.

\section{Fishbein, M., \& Ajzen, I.}

2010

Predicting and Changing Behavior: The Reasoned Action Approach. Nueva York: Psychology Press.

Folta, T., Delmar, F., \&

\section{Wennberg, $\mathrm{K}$.}

2010 Hybrid Entrepreneurship. Management Science, 56(2), 253-269. https://doi. org/10.1287/mnsc. 1090.1094

\section{Gilson, L., \& Goldberg, C.}

2015 Editors's comment: so, what is a conceptual paper? Group and Organization Management, 40(2), 127-130. https://doi. org/10.1177\%2F1059601115576425

\section{Guerra, G., \& Patuelli, R.}

The Role of Job Satisfaction in Transitions into Self-Employment. Entrepreneurship Theory and Practice, 40(3), 543-571. https:// doi.org/10.1111/etap.12133

Hausknecht, J., Rodda, J., \& Howard, $M$. 


\section{bibliografía}

reported reasons for staying. Human

Resource Management, 48(2), 269-288.

https://doi.org/10.1002/hrm.20279

\section{Hellmann, T.}

2007

When Do Employees Become

Entrepreneurs? Management Science,

21(5), 610-632. https://doi.org/10.1016/j.

jbusvent.2005.02.011

Hui-Chen, C., Kuen-Hung, T., \& Chen-Yi, P.

2014

The entrepreneurial process: an integrated model. International Entrepreneurship and Management Journal, (10), 727-745. https:// doi.org/10.1007/s11365-014-0305-8

\section{Hunt, S., \& Morgan, R.}

Organizational Commitment: One of Many Commitments or Key Mediating Construct? The Academy of Management Journal, 37(6), 1568-1587 https://doi. org/10.2307/256799.

\section{Kalleberg, A.}

Nonstandard Employment Relations: Parttime, temporary and contract work. Annual Review of Sociology, 26(1), 341-365 https:// doi.org/10.1146/annurev.soc.26.1.341

Klotz, A., Swider, B., Shao, Y., \& Prengler, $M$.

The paths from insider to outsider: A review of employee exit transitions. Human Resource Management, (60), 119-144. https://doi.org/10.1002/hrm.22033

\section{Kolvereid, L., \& Isaksen, E.}

New business start-up and subsequent entry into self-employment. Journal of Business Venturing, 21(6), 866-885. https:// doi.org/10.1016/j.jbusvent.2005.06.008
Kritskaya, L., Kolvereid, L., \& Isaksen, E.

2017 Hybrid entrepreneurs: characteristics and achievements. Entreprendre \& Innover, 34(3), 7-19. https://doi.org/10.3917/ entin.034.0007

Krueger, J. N., \& Carsrud, A. L.

Entrepreneurial intentions: Applying the theory of planned behaviour. Entrepreneurship \& Regional Development, 5(4), 315-330. https://doi. org/10.1080/08985629300000020

Krueger, N. F., Reilly, M. D., \& Carsrud, A. L.

Competing models of entrepreneurial intentions. Journal of Business Venturing, 15(5-6), 411-432. https://doi.org/10.1016/ s0883-9026(98)00033-0

\section{Krueger, N., \& Brazeal, D.}

Entrepreneurial Potential and Potential Entrepreneurs. Entrepreneurship Theory and Practice, 18(3), 91-104. https://doi. org/10.1177/104225879401800307

\section{Landgraf, A.}

Part-time entrepreneurship: Micro-level and macro-level determinants. Universität Trier. http://ubt.opus.hbz-nrw.de/ volltexte/2015/946/

Levie, J., \& Autio, E.

2013 Growth and Growth Intentions: A metaanalysis of existing evidence. Londres: Enterprise Research Centre.

\section{Liñan, F., \& Santos, F. J.}

Does Social Capital Affect Entrepreneurial Intentions? International Advances in 


\section{bibliografía}

Economic Research, (13), 443-453. https:// doi.org/10.1007/s11294-007-9109-8

\section{Liñán, F., Santos, F. J., \&}

\section{Fernández, J.}

2011

The influence of perceptions on potential entrepreneurs. International Entrepreneurship and Management Journal, (7), 373-390. https://doi. org/10.1007/s11365-011-0199-7

Luc, S., Chirita, G. M., Delvaux, E., \& Kepnou, A. K.

2018 Hybrid Entrepreneurship: Employees Climbing the Entrepreneurial Ladder. International Review of Entrepreneurship, 16(1), 89-114. https://www.senatehall.com/ entrepreneurship?article $=592$

\section{Marks, D., \& Yardley, L.}

2004 Research Methods for Clinical and Health Psychology. Londres: Sage.

\section{Marshall, D. R. \& Gigliotti, R.}

2018 Bound for entrepreneurship? A careertheoretical perspective on entrepreneurial intentions. International Entrepreneurship and Management Journal, (16), 287-303. https://doi.org/10.1007/s11365-018-0523-6

\section{Marshall, D.}

Work Conflicting With...Work? Examining the Effects of Running a Side Business on Employee Work and Entrepreneurial Venture Roles. En Entrepreneurship inside and out: Three essays exploring the interplay between hybrid entrepreneurs and their organizational employee roles (pp. 108-164). Electronic Theses and Dissertations. https://egrove.olemiss.edu/ etd/1358
Marshall, D., Davis, W., Dibrell,

\section{C., \& Ammeter, A.}

2019 Learning off the Job: Examining Part-time Entrepreneurs as Innovative Employees. Journal of Management, 45(8), 3091-3113. https://doi.org/10.1177/0149206318779127

\section{McClelland, D.}

The Achieving Society. Nueva Jersey: Van Nostrand.

\section{Meyer, J., \& Allen, N.}

A three-component conceptualization of organizational commitment. Human Resource Management Review, 1(1), 61-89. https://doi.org/10.1016/10534822(91)90011-Z

\section{Meyer, J., Becker, T., \&} Vandenberghe, $\mathrm{C}$.

Employee Commitment and Motivation: A Conceptual Analysis and Integrative Model. Journal of Applied Psychology, 89(1), 991-1007. https://doi.org/10.1037/00219010.89.6.991

\section{Miner, J., \& Dachler, P.}

1973 Personnel Attitudes and Motivation. Annual Review of Psychology, 24(1), 379-402. https://doi.org/10.1146/annurev. ps.24.020173.002115

Mourmant, G., \& Kalika, M. J.

Another road to IT turnover: the entrepreneurial path. European Journal of Information Systems, 18(5), 498-521. https://doi.org/10.1057/ejis.2009.37

Mowday, R. T., Steers, R. M., \& Porter, L. W. 


\section{bibliografía}

Behavior, 14(2), 224-247. https://doi

org/10.1016/0001-8791(79)90072-1

Neneh, B.

2020

Entrepreneurial self-efficacy and a

student's predisposition to choose an

entrepreneurial career path: the role of

self-perceived employability. Education

+ Training, 62(5), 559-580. https://doi.

org/10.1108/ET-06-2019-0108

\section{Popiel, P.}

2017

Boundaryless in the creative economy: assessing freelancing on upwork. Critical Studies in Media Communication, 34(3), 1-14. https://doi.org/10.1080/15295036.20 17.1282618

Porter, L., Steers, R., \& Mowday, R.

Organizational Commitment, Job

Satisfaction and Turnover among

Psychiatric Technicians. Journal of Applied

Psychology, 59(5), 603-609. https://doi.

org/10.1037/h0037335.

\section{Raffie, J., \& Feng, J.}

Should I Quit My Day Job?: A Hybrid

Path to Entrepreneurship. Academy of Management Journal, 57(4), 936-963.

https://doi.org/10.5465/amj.2012.0522

Ryan, R., \& Deci, E.

Self-determination Theory: When Mind Mediates Behavior. The Journal of Mind and Behavior, 1(1), 33-43. https://www. jstor.org/stable/43852807

\section{Schlaegel, C., \& Koenig, M.}

Theory and Practice, 38(2), 291-332.

https://doi.org/10.1111/etap.12087

\section{Schulz, M., Urbig, D., \& Procher, V.}

2016

Hybrid entrepreneurship and public policy:

The case of firm entry deregulation.

Journal of Business Venturing, 31(3),

272-286. https://doi.org/10.1016/j.

jbusvent.2016.01.002

\section{Shapero, A., \& Sokol, L.}

The Social Dimensions of Entrepreneurship.

En C. A. Kent, D. L. Sexton, \& K. H. Vesper (eds.), Encyclopedia of Entrepreneurship (pp. 72-90). Englewood Cliffs, Nueva Jersey: Prentice-Hal. https://ssrn.com/ abstract $=1497759$

\section{Solesvik, M.} Hybrid Entrepreneurship: How and Why Entrepreneurs Combine Employment with Self-Employment. Technology Innovation Management Review, 7(3), 33-41. https:// timreview.ca/article/1063

\section{Solinger, O., Van Olffen, W., \&} Roe, R.

Beyond the Three-Component Model of Organizational Commitment. Journal of Applied Psychology, 93(1), 70-83. https:// doi.org/10.1037/0021-9010.93.1.70

Sørensen, J. B., \& Fassiotto, M. A. 2011 Organizations as Fonts of Entrepreneurship. Organization Science, 22(5), 1121-1367. https://doi.org/10.1287/orsc. 1100.0622

Sun, J., Huang, Y., Su, D., \& Yang, $\mathbf{C}$.

entrepreneurs from the perspective 


\section{bibliografía}

of entrepreneurial ability. Information

Systems and e-Business Management.

18(3), 455-484. https://doi.org/10.1007/

s10257-019-004

\section{Tett, R., \& Meyer, J.}

Job Satisfaction, Organizational

Commitment, Turnover Intention

and Turnover: Path analysis based

on metanalytic findings. Personnel

Psychology, 46(2), 259-293. https://doi.

org/10.1111/j.1744-6570.1993.tb00874.x

Thorgren, S., Nordström, C., \& Wincent, J.

Hybrid entrepreneurship: The importance of passion. Baltic Journal Of Management, 9(3) http://dx.doi.org/10.1108/BJM-11-2013-0175

\section{Thorgren, S., Sirén, C.,}

\section{Nordström, C., \& Wincent, J.}

Hybrid entrepreneurs' second-step

choice: The nonlinear relationship between age and intention to enter full-time entrepreneurship. Journal of Business Venturing Insights, $5(\mathrm{C})$,

14-18. https://EconPapers.repec.org/

RePEc:eee:jobuve:v:5:y:2016:i:c:p:14-18

\section{Van der Zwan, P., \& Thurik, R.}

Entrepreneurship as a process: Emprirical Evidence for Entrepreneurial Engagement Levels. En G. Ahmetoglu, T. ChamorroPremuzic, B. Klinger y T. Karcisky (eds.), The Wiley Handbook of Entrepreneurship (pp. 25-33). John Wiley \& Sons Ltd

\section{Viljamaa, A., \& Varamäki, E.}

An Interconnected World: Pioneering Business And Technology Excellence (pp. 635-642). http://gbata.org/wp-content/ uploads/2014/08/GBATA2014-ReadingsBook.pdf

\section{Viljamaa, A., \& Varamäki, E.}

Best of Both Worlds? Persistent Hybrid Entrepreneruship. Journal of Enterprising Culture, 25(4), 339-359. https://doi. org/10.1142/S0218495817500133

Viljamaa, A., Varamäki, E., Tornikoski, E., \& Sorama, K.

Hybrid Entrepreneurship - Exploration of Motives, Ambitions and Growth. En ICSB World Conference Proceedings (pp. 1-3). Washington, D. C.: International Council for Small Business.

Von Neumann, J., \& Morgenstern, $\mathbf{O}$.

Theory of Games and Economic Behavior. Princeton: Princeton University Press.

\section{Vroom, V.} Work and Motivation. Nueva York: Wiley and Sons.

\section{Vukovic, \& Kedmenec.}

The role of bonding and bridging social capital in shaping entrepreneurial intention in transition economies. Management: Journal or Contemporary Management Issues, 22(1), 1-33. https://doi. org/10.30924/MJCMI/2017.22.1.1

\section{Wagner, J.}

2004 Nascent Entrepreneurs. IZA DP, (1293), 1-33. 


\section{bibliografía}

\section{Weick, K.}

1989 Theory construction as disciplined

imagination. Academy of Management

Review, 14(4), 516-531. https://doi.

org/10.2307/258556

\section{Westlund, H., \& Bolton, R.}

2003 Local Social Capital and Entrepreneurship. Small Business Economics, (21), 77-113.

https://doi.org/10.1023/A:1025024009072

Zambrano, S., Zambrano, Y., \&

Chávez, A.

2020 Dimensiones para el estudio de la

intención emprendedora en jóvenes

universitarios. Revista Espacios, 41(20).

http://www.revistaespacios.com/

a20v41n20/a20v41n20p28.pdf

Zhao, H., Seibert, C., \& Hilla, C.

2005 The mediating role of self-efficacy in the development of entrepreneurial intentions. Journal of Applied Psychology, 90(6), 1265-1272. https://doi.org/10.1037/00219010.90.6.1265

Fecha de recepción: 16 de septiembre de 2021 Fecha de aprobación: 4 de noviembre de 2021 Fecha de publicación: 20 de diciembre de 2021 